\title{
UTILITY BASED OPTIMAL HEDGING IN INCOMPLETE MARKETS ${ }^{1}$
}

\author{
BY M. P. OWEN \\ Vienna University of Technology
}

\begin{abstract}
We provide the solution to a fusion of two fundamental problems in mathematical finance. The first problem is that of maximizing the expected utility of terminal wealth of an investor who holds a short position in a contingent claim, and the second is that of maximizing terminal wealth where the utility function allows the investor to have negative wealth. Under assumptions of reasonable asymptotic elasticity on the investor's utility function, we present an optimal investment theorem and simultaneously treat the corresponding dual problem.
\end{abstract}

1. Introduction and statement of results. This paper addresses the problem of maximizing the expected utility of terminal wealth of an investor who holds a short position in a contingent claim. Portfolio optimization for an investor whose preferences are expressed in terms of a utility function is a classical theme in mathematical finance. An account of advances concerning this theme can be found in [13].

For recent articles in the area of utility maximization, see [10, 14, 1, 3, 5], to cite just a few. The article [10] solves the optimal portfolio problem for the case of utility functions supporting positive wealths in the general framework of a semimartingale market model. This article was subsequently extended in [1], to cover the case of an investor who additionally receives a random endowment $e_{T}$ at the end of trading. The situation of receiving a random endowment is of course no different to that of honoring a short position in a contingent claim $B=-e_{T}$ at time $T$. The articles $[14,5]$ extend [10] in a different direction, allowing the investor the possibility of being in debt at the end of trading in order to maximize their expected utility.

In the article [3], the settings of [1] and [14] are partially brought together: They treat the case of an investor who essentially wishes to hedge a contingent claim by maximising expected exponential utility. Their analysis is valid for contingent claims which are bounded below, and which satisfy a mild condition above, which is weakly related to superhedgability of the claim. In this article, we solve the same problem for more general utility functions supporting positive and negative wealths, where the short position is in a bounded claim. We assume that the utility function has reasonable asymptotic elasticity, as introduced in [10] and [14].

Received April 2001.

${ }^{1}$ Supported by the Austrian Science Foundation Wittgenstein-Prize Grant Z36-MAT. AMS 2000 subject classifications. Primary 91B28, 91B16.

Key words and phrases. Utility, hedging, incomplete markets, contingent claims. 
Let $U: \mathbb{R} \rightarrow \mathbb{R}$ be a strictly concave, continuously differentiable function satisfying

$$
U^{\prime}(-\infty)=\infty, \quad U^{\prime}(\infty)=0 .
$$

The conditions for reasonable asymptotic elasticity are

$$
\operatorname{AE}_{-\infty}(U):=\liminf _{x \rightarrow-\infty} \frac{x U^{\prime}(x)}{U(x)}>1
$$

and

$$
\mathrm{AE}_{+\infty}(U):=\limsup _{x \rightarrow \infty} \frac{x U^{\prime}(x)}{U(x)}<1 .
$$

Some equivalent conditions to reasonable asymptotic elasticity can be found in [14].

We model the discounted price process of $d$ risky assets as a $d$-dimensional, locally bounded semimartingale $S=\left(\left(S_{t}^{i}\right)_{1 \leq i \leq d}\right)_{0 \leq t \leq T}$. The financial market also comprises a riskless asset whose discounted price $B_{t} \equiv 1$. The semimartingale is based on a filtered probability space $\left(\Omega, \mathcal{F},\left(\mathcal{F}_{t}\right)_{0 \leq t \leq T}, \mathbb{P}\right)$, where the filtration $\left(\mathcal{F}_{t}\right)_{0 \leq t \leq T}$ is assumed to satisfy the usual conditions of right continuity and completeness, and the time horizon $T$ is finite.

The family of equivalent (resp. absolutely continuous) local martingale measures will be denoted by $\mathcal{M}^{e}(S)$ (resp. $\left.\mathcal{M}^{a}(S)\right)$. We assume throughout this paper that $\mathcal{M}^{e}(S) \neq 0$. This condition is intimately related to the absence of arbitrage opportunities. See [10] and [4] for detailed discussions on this subject.

A contingent claim $B$ at time $T$ is an $\mathcal{F}_{T}$-measurable random variable. This article treats the case where an investor is short such a claim. Due to some technical considerations, we shall assume throughout that $\|B\|_{\infty}<\infty$ [see, however, Remark 1.2(ii)]. It should be possible to relax this assumption a certain amount in future work.

Our aim is to study the optimization problem

$$
u(x):=\sup _{X \in \mathcal{X}_{U}(x)} \mathbb{E}[U(x+X-B)]
$$

and to formulate a duality result. In order to rule out trivial cases, we shall throughout the article make the assumption that

$$
u(x)<U(\infty) \quad \text { for some } x \in \mathbb{R} .
$$

In formula (2) above, we make the crucial choice

$$
\begin{aligned}
& \mathcal{X}_{U}(x):=\left\{X \in L^{0}\left(\Omega, \mathcal{F}_{T}, \mathbb{P} ; \mathbb{R} \cup\{\infty\}\right):\right. \\
& \left.U(x+X-B) \in{\overline{\left\{U(F): F \in \mathcal{F}_{U}^{b}(x)\right.}}^{L^{1}(\mathbb{P})}\right\},
\end{aligned}
$$


where

$$
\mathcal{F}_{U}^{b}(x):=\left\{F \in L^{0}\left(\Omega, \mathcal{F}_{T}, \mathbb{P}\right): F \leq x+(H \cdot S)_{T}-B\right.
$$

for some admissible portfolio $H$, and $\left.U(F) \in L^{1}(\mathbb{P})\right\}$.

The admissible portfolios in (5) are those predictable $S$-integrable processes $H$ for which the stochastic integral $(H \cdot S)_{t}=\int_{0}^{t} H_{u} \mathrm{~d} S_{u}$ is $\mathbb{P}$-a.s. uniformly bounded from below.

The set $\mathcal{X}_{U}(x)$ is chosen large enough to contain an optimal solution, and small enough so that

$$
\sup _{X \in \mathcal{F}_{U}^{b}(x)} \mathbb{E}[U(x+X-B)]=\sup _{X \in \mathcal{X}_{U}(x)} \mathbb{E}[U(x+X-B)] .
$$

As in [14], our solution of the primal problem (2) relies upon first solving the dual minimization problem

$$
v(y)=\inf _{\mathbb{Q} \in \mathcal{M}^{a}(S)} \mathbb{E}_{\mathbb{P}}\left[V\left(y \frac{\mathrm{d} \mathbb{Q}}{\mathrm{dP}}\right)-y \frac{\mathrm{d} \mathbb{Q}}{\mathrm{dP}} B\right],
$$

where we now have an additional term due to the contingent claim. Here, the conjugate function $V(y)$ of the utility function $U(x)$ is defined by

$$
V(y)=\sup _{x \in \mathbb{R}}[U(x)-x y], \quad y>0,
$$

which, under the conditions (1), is a continuously differentiable, convex function satisfying

$$
V(0)=U(\infty), \quad V(\infty)=\infty, \quad V^{\prime}(0)=-\infty, \quad V^{\prime}(\infty)=\infty .
$$

It is simple to verify that

$$
V(y)=U(I(y))-y I(y),
$$

where $I(y):=-V^{\prime}(y)=\left(U^{\prime}\right)^{-1}(y)$.

We are now able to state the main result:

THEOREM 1.1. Assume that $U$ satisfies condition (1) and has reasonable asymptotic elasticity, and that (3) is also satisfied. Let B be a bounded contingent claim. Then:

(i) The value functions $u$ and $v$ are finitely valued, strictly concave (resp. convex), continuously differentiable functions defined on $\mathbb{R}$ (resp. $\left.\mathbb{R}_{+}\right)$; they are conjugate and satisfy

$$
u^{\prime}(\infty)=0, \quad u^{\prime}(-\infty)=\infty, \quad v^{\prime}(0)=-\infty, \quad v^{\prime}(\infty)=\infty .
$$

The value function $u$ has reasonable asymptotic elasticity.

(ii) For $y>0$, the optimal solution $\mathbb{Q}_{y} \in \mathcal{M}^{a}(S)$ to the dual problem (6) exists, is unique, and the map $y \mapsto \mathbb{Q}_{y}$ is continuous in the variation norm. 
(iii) For $x \in \mathbb{R}$ the optimal solution $\hat{X}(x)$ to the primal problem (2) exists, is unique, and satisfies

$$
x+\hat{X}(x)-B=-V^{\prime}\left(y \frac{\mathrm{d} \mathbb{Q}_{y}}{\mathrm{~d} \mathbb{P}}\right),
$$

where $y=u^{\prime}(x)$.

(iv) We have the formulae

$$
\begin{aligned}
v^{\prime}(y) & =\mathbb{E}_{\mathbb{Q}_{y}}\left[V^{\prime}\left(y \frac{\mathrm{d} \mathbb{Q}_{y}}{\mathrm{dP}}\right)-B\right], \\
u^{\prime}(x) & =\mathbb{E}_{\mathbb{P}}\left[U^{\prime}(x+\hat{X}(x)-B)\right], \\
x u^{\prime}(x) & =\mathbb{E}_{\mathbb{P}}\left[(x+\hat{X}(x)) U^{\prime}(x+\hat{X}(x)-B)\right],
\end{aligned}
$$

where the usual rule $\infty \cdot 0=0$ is applied, if the integrands are irregular.

(v) If $\mathbb{Q}_{y} \in \mathcal{M}^{e}(S)$ where $y=u^{\prime}(x)$, then $\hat{X}(x)$ is the terminal value of a process of the form $\hat{X}_{t}(x)=(\hat{H} \cdot S)_{t}$, where $\hat{H}$ is predictable and $S$-integrable, such that $\hat{X}_{t}$ is a uniformly integrable martingale under $\mathbb{Q}_{y}$.

REMARKS 1.2. (i) The case of a complete market, where there is a unique local martingale measure $\mathbb{Q}$, is of course covered by the above theorem. However, much of the analysis required to prove the theorem in this situation is redundant; the dual problem is trivially optimized by $\mathbb{Q}$, and it remains only to prove that

$$
X=-x-V^{\prime}\left(y \frac{\mathrm{d} \mathbb{Q}}{\mathrm{dP}}\right)+B
$$

is the unique optimal solution to the primal problem.

(ii) Although this article only treats the case of bounded claims (e.g., European puts), we can quite easily extend our result to cover European calls: Let $S$ be a 1-dimensional, non-negative semimartingale, and assume that the set $\mathbb{M}^{e}(S)$ of equivalent martingale measures is non-empty. We extend our definition of admissible portfolios slightly, and say that a portfolio $H$ is $B$-admissible if

$$
(H \cdot S)_{t}-\mathbb{E}_{\mathbb{Q}}\left[B \mid \mathcal{F}_{t}\right]
$$

is $\mathbb{P}$-a.s. uniformly bounded below for each $\mathbb{Q} \in \mathbb{M}^{e}(S)$. We define $\mathcal{X}_{U, B}(x)$ the same way as $\mathcal{X}_{U}(x)$, except using $B$-admissible processes in place of admissible processes. If $B$ is bounded, $B$-admissibility reduces to normal admissibility. However, if $B$ is unbounded, we have the more appropriate economic interpretation that under every martingale (pricing) measure, the investor's discounted total worth is uniformly bounded below.

Let $B=\left(S_{T}-K\right)^{+}$be the payoff of a European call option for some strike price $K>0$. The claim $B^{\prime}:=B-S_{T}$ is bounded, and therefore the optimization problem

$$
u(x)=\sup _{X^{\prime} \in \mathcal{X}_{U, B^{\prime}}(x)} \mathbb{E}\left[U\left(x+X^{\prime}-B^{\prime}\right)\right]
$$


has a unique solution $\hat{X}^{\prime}$. Defining $\hat{X}=\hat{X}^{\prime}-S_{T}$, we see that $\hat{X}$ is the optimal solution of the problem

$$
u(x)=\sup _{X \in X_{U, B}(x)} \mathbb{E}[U(x+X-B)] .
$$

To see this we simply need to show that $X \in \mathcal{X}_{U, B}(x)$ if and only if $X^{\prime}:=$ $X-S_{T} \in \mathcal{X}_{U, B^{\prime}}(x)$. In fact, it suffices to show that $H$ is $B$-admissible if and only if $H^{\prime}:=H-1$ is admissible. This follows easily from the martingale property of $S$ under all $\mathbb{Q} \in \mathbb{M}^{e}(S)$.

(iii) Optimal hedging in incomplete markets plays a role in determining a fair price for a contingent claim. The technique of marginal rate of substitution (see, e.g., [2]) chooses a price such that substituting a marginal amount of a claim for the same fraction of the price has a neutral effect on the investor's achievable expected utility. A similar approach is adopted in [8] for utility functions which allow negative wealth. They require that the price $p$ satisfies

$$
\sup _{X \in X_{U, 0}(x+p)} \mathbb{E}[U(x+X+p)]=\sup _{X \in \mathcal{X}_{U, B}(x)} \mathbb{E}[U(x+X+B)] .
$$

El Karoui and Rouge pay special attention to the case of bounded claims and exponential utility.

2. Approximating the utility function by limiting credit. Given the utility function $U$ of Theorem 1.1, we shall take the approach used in [14] of approximating $U$ from below by an increasing sequence of utility functions with credit limits which become gradually less restrictive to the investor. We fix the approximating utility functions $U_{n}$ to be identically equal to $U$ on the interval $[-n, \infty)$ and require them to be strictly concave, continuously differentiable, and to satisfy $U_{n}(x)=-\infty$ for $x<-(n+1)$, and $U_{n}(x)>-\infty$ for $x>-(n+1)$. We also assume that

$$
\lim _{x \searrow-(n+1)} U_{n}^{\prime}(x)=\infty
$$

holds for each of the approximating functions.

It is not difficult to show that there exists a constant $C$ such that the estimates of [14, Corollary 4.2] are simultaneously valid for $V$ and all $V_{n}$ (i.e., for a constant $C$ independent of $n$ ).

Using a minor modification of the analysis of [1] [shifting the singularity of $U_{n}$ at $x=-(n+1)$ to 0 , applying their result and then shifting back again], we see that the optimization problem

$$
u_{n}(x)=\sup _{X \in X_{0}} \mathbb{E}\left[U_{n}(x+X-B)\right],
$$

$$
\mathcal{X}_{0}:=\left\{X: X \leq(H \cdot S)_{T} \text { for some admissible portfolio } H\right\},
$$

has a unique optimal solution $\hat{X}^{n}(x) \in \mathcal{X}_{0}$ for $x>\rho-(n+1)$, where

$$
\rho:=\sup _{\mathbb{Q} \in \mathcal{M}^{e}(S)} \mathbb{E}_{\mathbb{Q}}[B] \leq\left\|B^{+}\right\|_{\infty}<\infty .
$$


(In the case of $S$ bounded below, $\rho$ is the price of superhedging $B$; see, e.g., [7, 9] and [6], Remark 2.2.) Their analysis shows that the dual problem

$$
\begin{aligned}
v_{n}(y)= & \inf _{\mathbb{Q} \in \mathscr{D}}\left\{\mathbb{E}\left[V_{n}\left(y \frac{\mathrm{d} \mathbb{Q}^{r}}{\mathrm{~d} \mathbb{P}}\right)\right]+y(n+1)\left(1-\mathbb{E}\left[\frac{\mathrm{d} \mathbb{Q}^{r}}{\mathrm{~d} \mathbb{P}}\right]\right)-y\langle\mathbb{Q}, B\rangle\right\}, \\
\mathscr{D}:= & \left\{\mathbb{Q} \in L^{\infty}\left(\Omega, \mathcal{F}_{T}, \mathbb{P}\right)^{*}:\|\mathbb{Q}\|=1\right. \\
& \text { and } \left.\langle\mathbb{Q}, X\rangle \leq 0 \text { for all } X \in \mathcal{X}_{0} \cap L^{\infty}\right\}
\end{aligned}
$$

to (9) has a unique optimal solution $\mathbb{Q}_{n, y} \in \mathscr{D}$. Here $V_{n}$ is the conjugate function of $U_{n}$. It is proved in [1] that $v_{n}$ is conjugate to $u_{n}$, that for any $n \in \mathbb{N}$ and any $x, y$ satisfying $y=u_{n}^{\prime}(x)$ [or alternatively $x=-v_{n}^{\prime}(y)$ ] we have the relation

$$
x+\hat{X}^{n}(x)-B=-V_{n}^{\prime}\left(y \frac{\mathrm{d} \mathbb{Q}_{n, y}^{r}}{\mathrm{~d} \mathbb{P}}\right),
$$

and that

$$
\text { (15) } v_{n}^{\prime}(y)=\mathbb{E}\left[\frac{\mathrm{d} \mathbb{Q}_{n, y}^{r}}{\mathrm{~d} \mathbb{P}} V_{n}^{\prime}\left(y \frac{\mathrm{d} \mathbb{Q}_{n, y}^{r}}{\mathrm{~d} \mathbb{P}}\right)\right]+(n+1)\left(1-\mathbb{E}\left[\frac{\mathrm{d} \mathbb{Q}_{n, y}^{r}}{\mathrm{~d} \mathbb{P}}\right]\right)-\langle\mathbb{Q}, B\rangle .
$$

Throughout this article we assume without loss of generality that $U(0)>0$ so that $V(y)>0$ and $V_{n}(y)>0$ for all $y>0$ and $n \in \mathbb{N}$.

REMARKS 2.1. (i) The set $\mathscr{D}$ is convex and compact in the weak* topology on $L^{\infty}\left(\Omega, \mathcal{F}_{T}, \mathbb{P}\right)^{*}$. This can be seen by applying Alaoğlu's Theorem (see, e.g., [11], Theorem IV.1.4).

(ii) Note that

$$
\langle\mathbb{Q}, X\rangle \leq 0
$$

for all $\mathbb{Q} \in \mathscr{D}$ and all $X \in \mathcal{X}_{0}$ which are uniformly bounded from below (actually, (16) holds for all $\mathbb{Q} \in \mathscr{D}, X \in \mathcal{X}_{0}$ ). See [1], Section 3.

(iii) Using the canonical embedding of $L^{1}(\mathbb{P})$ in $\left(L^{\infty}(\mathbb{P})\right)^{*}$, note that $\mathscr{D} \cap L^{1}=$ $\mathcal{M}^{a}(S)$. To see this, we use the fact that $S$ is locally bounded to obtain a sequence of stopping times $\left(\tau_{n}\right)_{n=1}^{\infty}$ such that $\tau_{n} \rightarrow \infty$ a.s., and such that $S^{\tau_{n}}$ is bounded for each $n$. For each $n, S^{\tau_{n}},-S^{\tau_{n}} \in \mathcal{X}_{0} \cap L^{\infty}$, and therefore $\mathbb{E}\left[\frac{\mathrm{d} \mathbb{Q}}{\mathrm{d} \mathbb{P}} S^{\tau_{n}}\right]=0$ for all $\mathbb{Q} \in \mathscr{D} \cap L^{\infty}$ by the definition of $\mathscr{D}$. This is enough to show that $S^{\tau_{n}} Z$ is a martingale, where $Z_{t}:=\mathbb{E}\left[\frac{\mathrm{d} \mathbb{Q}}{\mathrm{dP}} \mid \mathcal{F}_{t}\right]$, and therefore $S$ is a local martingale under $\mathbb{Q}$.

(iv) For each $\mathbb{Q} \in\left(L^{\infty}(\mathbb{P})\right)^{*}$, there is a unique decomposition $\mathbb{Q}=\mathbb{Q}^{r}+\mathbb{Q}^{s}$ of $\mathbb{Q}$ into its regular and singular parts, where the regular part is countably additive and absolutely continuous with respect to $\mathbb{P}$ on the sigma algebra $\mathcal{F}$ modulo the nullsets, and the singular part is purely finitely additive. 
It is easy to show that for fixed $y, v_{n}(y)$ is monotone increasing and bounded above by $v(y)$. We define now the function

$$
v_{\infty}(y):=\sup _{n \in \mathbb{N}} v_{n}(y),
$$

which turns out later to be the function $v$. The following simple lemma will be useful:

\section{LEMMA 2.2. The function $v_{\infty}$ is finitely valued and dominated by $v$.}

PROOF. Condition (3) requires $u(x)$ to be finite for some (actually all) $x \in \mathbb{R}$, and therefore the conjugate function $u^{*}$ of $u$ must be finite for some $\bar{y}$. Thus

$$
v_{n}(\bar{y})=\sup _{x \in \mathbb{R}}\left[u_{n}(x)-x \bar{y}\right] \leq \sup _{x \in \mathbb{R}}[u(x)-x \bar{y}]=u^{*}(\bar{y}) .
$$

Using equation (12) and [14], Corollary 4.2(i), we see that for $y>0$ there exists a constant $C=C(y / \bar{y})$ such that for $n$ large enough,

$$
\begin{aligned}
v_{n}(y) \leq & \mathbb{E}\left[V_{n}\left(y \frac{\mathrm{d} \mathbb{Q}_{n, \bar{y}}^{r}}{\mathrm{~d} \mathbb{P}}\right)\right]+y(n+1)\left(1-\mathbb{E}\left[\frac{\mathrm{d} \mathbb{Q}_{n, \bar{y}}^{r}}{\mathrm{~d} \mathbb{P}}\right]\right)-y\left\langle\mathbb{Q}_{n, \bar{y}}, B\right\rangle \\
\leq & C \mathbb{E}\left[V_{n}\left(\bar{y} \frac{\mathrm{d} \mathbb{Q}_{n, \bar{y}}^{r}}{\mathrm{~d} \mathbb{P}}\right)\right] \\
& +\frac{y}{\bar{y}}\left(\bar{y}(n+1)\left(1-\mathbb{E}\left[\frac{\mathrm{d} \mathbb{Q}_{n, \bar{y}}^{r}}{\mathrm{~d} \mathbb{P}}\right]\right)-\bar{y}\left\langle\mathbb{Q}_{n, \bar{y}}, B\right\rangle+\bar{y}\left\|B^{+}\right\|_{\infty}\right) \\
\leq & \max \{C, y / \bar{y}\}\left(v_{n}(\bar{y})+\bar{y}\left\|B^{+}\right\|_{\infty}\right) \\
\leq & \max \{C, y / \bar{y}\}\left(u^{*}(\bar{y})+\bar{y}\left\|B^{+}\right\|_{\infty}\right)<\infty .
\end{aligned}
$$

To show that $v_{\infty}$ is dominated by $v$, just note that the set $\mathscr{D}$, over which the infimum for $v_{n}$ in (12) is taken, contains the set $\mathcal{M}^{a}(S)$, over which the infimum for $v$ in (6) is taken.

Since the sequence $\left(v_{n}\right)_{n=1}^{\infty}$ increases monotonely to the limiting function $v_{\infty}$, the following lemma will also be useful:

LEMMA 2.3. Suppose that $v_{n}$ is a sequence of convex (or concave) functions which increases (or decreases) monotonely pointwise to a convex (resp. concave) function $v_{\infty}$, and let $\left(y_{n}\right)_{n=1}^{\infty}$ be a sequence of real numbers tending to $y$ in the domain of $v_{\infty}$. Then $v_{n}\left(y_{n}\right) \rightarrow v_{\infty}(y)$ as $n \rightarrow \infty$ and, provided the derivatives exist, $v_{n}^{\prime}\left(y_{n}\right) \rightarrow v_{\infty}^{\prime}(y)$ as $n \rightarrow \infty$. 
PROOF. The statement that $v_{n}\left(y_{n}\right) \rightarrow v_{\infty}(y)$ is well known as Dini's theorem (see, e.g., [11], Theorem IX.1.3). The convergence of $v_{n}^{\prime}\left(y_{n}\right)$ to $v^{\prime}(y)$ follows easily from [12], Theorem 25.7, where it is shown that the derivatives $v_{n}^{\prime}$ converge uniformly to $v_{\infty}^{\prime}$ on closed bounded intervals.

3. Proofs. The proof of Theorem 1.1 will be broken up into Lemma 3.1, Corollary 3.2 and Lemma 3.3.

LEMMA 3.1. Let $\left(y_{n}\right)_{n=1}^{\infty}$ be a sequence of positive real numbers tending to $y$. Then there exists a $\mathbb{Q}_{y} \in \mathcal{M}^{a}(S)$ such that:

(i)

$$
\mathbb{Q}_{n, y_{n}} \stackrel{L^{\infty}(\mathbb{P})^{*}}{\longrightarrow} \mathbb{Q}_{y}
$$

(ii)

$$
V_{n}\left(y_{n} \frac{\mathrm{d} \mathbb{Q}_{n, y_{n}}^{r}}{\mathrm{~d} \mathbb{P}}\right) \stackrel{L^{1}(\mathbb{P})}{\longrightarrow} V\left(y \frac{\mathrm{d} \mathbb{Q}_{y}}{\mathrm{~d} \mathbb{P}}\right)
$$

(iii)

$$
y_{n} \frac{\mathrm{d} \mathbb{Q}_{n, y_{n}}^{r}}{\mathrm{~d} \mathbb{P}} V_{n}^{\prime}\left(y_{n} \frac{\mathrm{d} \mathbb{Q}_{n, y_{n}}^{r}}{\mathrm{~d} \mathbb{P}}\right) \stackrel{L^{1}(\mathbb{P})}{\longrightarrow} y \frac{\mathrm{d} \mathbb{Q}_{y}}{\mathrm{~d} \mathbb{P}} V^{\prime}\left(y \frac{\mathrm{d} \mathbb{Q}_{y}}{\mathrm{~d} \mathbb{P}}\right)
$$

(iv)

$$
y \frac{\mathrm{d} \mathbb{Q}_{y}}{\mathrm{~d} \mathbb{P}} V_{n}^{\prime}\left(y_{n} \frac{\mathrm{d} \mathbb{Q}_{n, y_{n}}}{\mathrm{~d} \mathbb{P}}\right) \stackrel{L^{1}(\mathbb{P})}{\longrightarrow} y \frac{\mathrm{d} \mathbb{Q}_{y}}{\mathrm{~d} \mathbb{P}} V^{\prime}\left(y \frac{\mathrm{d} \mathbb{Q}_{y}}{\mathrm{~d} \mathbb{P}}\right)
$$

$$
U\left(x+\hat{X}_{T}^{n}(x)-B\right) \stackrel{L^{1}(\mathbb{P})}{\longrightarrow} U(x+\hat{X}(x)-B),
$$

where $\hat{X}(x):=-\left(x+V^{\prime}\left(y \frac{\mathrm{d} \mathbb{Q}_{y}}{\mathrm{~d} \mathbb{P}}\right)-B\right)$.

In the following corollary, $\left(y_{n}\right)_{n=1}^{\infty}$ is still a sequence of strictly positive numbers tending to $y>0$.

COROLlaRY 3.2. (i) The map $y \mapsto \frac{\mathrm{d}_{y}}{\mathrm{~d} \mathbb{P}}$ is continuous in the $L^{1}(\mathbb{P})$ norm,

$$
v_{n}\left(y_{n}\right) \rightarrow v(y)=\mathbb{E}\left[V\left(y \frac{\mathrm{d} \mathbb{Q}_{y}}{\mathrm{dP}}\right)-y \frac{\mathrm{d} \mathbb{Q}_{y}}{\mathrm{dP}} B\right],
$$

and thus $\mathbb{Q}_{y}$ is the unique minimizer of the dual problem (6). The function $v$ is strictly convex.

(ii) The map $y \mapsto V\left(y \frac{\mathrm{d} \mathbb{Q}_{y}}{\mathrm{~d} \mathbb{P}}\right)$ is continuous in the $L^{1}(\mathbb{P})$ norm. 
(iii) The map $y \mapsto \frac{\mathrm{d}_{y}}{\mathrm{~d} \mathbb{P}} V^{\prime}\left(y \frac{\mathrm{d}_{y}}{\mathrm{~d} \mathbb{P}}\right)$ is continuous in the $L^{1}(\mathbb{P})$ norm. The function $v$ is continuously differentiable and

$$
v_{n}^{\prime}\left(y_{n}\right) \rightarrow v^{\prime}(y)=\mathbb{E}\left[\frac{\mathrm{d} \mathbb{Q}_{y}}{\mathrm{~d} \mathbb{P}}\left(V^{\prime}\left(y \frac{\mathrm{d} \mathbb{Q}_{y}}{\mathrm{~d} \mathbb{P}}\right)-B\right)\right] .
$$

(iv) Let $x=-v^{\prime}(y)$. Then

$$
\hat{X}_{T}^{n}(x) \stackrel{L^{1}\left(\mathbb{Q}_{y}\right)}{\longrightarrow} \hat{X}(x):=-\left(x+V^{\prime}\left(y \frac{\mathrm{d} \mathbb{Q}_{y}}{\mathrm{dP}}\right)-B\right) ;
$$

(v)

$$
u_{n}(x) \rightarrow u(x)=\mathbb{E}[U(x+\hat{X}(x)-B)]
$$

and $\hat{X}(x) \in \mathcal{X}_{U}(x)$, thus $\hat{X}(x)$ is the unique maximizer of the primal problem.

The function $u$ is conjugate to $v$, has reasonable asymptotic elasticity and satisfies the conditions

$$
\begin{gathered}
u^{\prime}(-\infty)=\infty, \quad u^{\prime}(\infty)=0, \\
u^{\prime}(x)=\mathbb{E}\left[U^{\prime}(x+\hat{X}(x)-B)\right], \\
x u^{\prime}(x)=\mathbb{E}\left[(x+\hat{X}(x)) U^{\prime}(x+\hat{X}(x)-B)\right] .
\end{gathered}
$$

PROOF OF LEMMA 3.1(i). We shall first show that the sequence $\left(\frac{\mathrm{d} \mathbb{Q}_{n, y_{n}}^{r}}{\mathrm{dP}}\right)_{n=1}^{\infty}$ converges in the norm of $L^{1}(P)$. To do this, we show that the sequence is uniformly integrable and Cauchy in the topology of convergence in probability.

Suppose for a contradiction that the sequence $\left(\frac{d \mathbb{Q}_{n, y_{n}}^{r}}{d r}\right)_{n=1}^{\infty}$ fails to be uniformly integrable, or equivalently that the sequence $\left(y_{n} \frac{\mathrm{d} \mathbb{Q}_{n, y_{n}}^{r}}{\mathrm{~d} \mathbb{P}}\right)_{n=1}^{\infty}$ fails to be uniformly integrable, that is, there is an $\alpha>0$ such that for each $C>0$,

$$
\limsup _{n \rightarrow \infty} \mathbb{E}\left[y_{n} \frac{\mathrm{d} \mathbb{Q}_{n, y_{n}}^{r}}{\mathrm{~d} \mathbb{P}} \chi_{\left\{y_{n} \frac{\mathrm{d} \mathbb{Q}_{n, y_{n}}^{r}}{\mathrm{~d} \mathbb{P}} \geq C\right\}}\right]>\alpha .
$$

It follows from the inequality

$$
V_{m}(z) \geq U_{m}(-m)+m z
$$

and the assumption $U_{m}(-m)>-\infty$ that

$$
\lim _{z \rightarrow \infty} \frac{V_{m}(z)}{z} \geq m
$$

Fix $m \in \mathbb{N}$, find $C_{m}$ such that $V_{m}(z) \geq(m-1) z$ for $z \geq C_{m}$, and find $n>m$ such that

$$
\mathbb{E}\left[y_{n} \frac{\mathrm{d} \mathbb{Q}_{n, y_{n}}^{r}}{\mathrm{~d} \mathbb{P}} \chi_{\left\{y_{n} \frac{\mathrm{d} \mathbb{Q}_{n, y_{n}}^{r}}{\mathrm{~d} \mathbb{P}} \geq C_{m}\right\}}\right]>\alpha .
$$


Using (12),

$$
\begin{aligned}
v_{n}\left(y_{n}\right) & \geq \mathbb{E}\left[V_{n}\left(y_{n} \frac{\left.\left.\mathrm{d} \mathbb{Q}_{n, y_{n}}^{r}\right)\right]-y_{n}\left\|B^{+}\right\|_{\infty}}{\mathrm{d} \mathbb{P}}\right)\right. \\
& \geq \mathbb{E}\left[V_{m}\left(y_{n} \frac{\mathrm{d} \mathbb{Q}_{n, y_{n}}^{r}}{\mathrm{~d} \mathbb{P}}\right) \chi_{\left\{y_{n} \frac{\mathrm{d} Q_{n, y_{n}}^{r}}{\mathrm{~d} \mathbb{P}} \geq C_{m}\right\}}\right]-y_{n}\left\|B^{+}\right\|_{\infty} \\
& \geq \mathbb{E}\left[(m-1) y_{n} \frac{\mathrm{d} \mathbb{Q}_{n, y_{n}}^{r}}{\mathrm{~d} \mathbb{P}} \chi_{\left\{y_{n} \frac{\mathrm{d}_{n, y_{n}}^{r}}{\mathrm{~d} \mathbb{P}} \geq C_{m}\right\}}\right]-y_{n}\left\|B^{+}\right\|_{\infty} \\
& \geq(m-1) \alpha-y_{n}\left\|B^{+}\right\|_{\infty},
\end{aligned}
$$

which contradicts the boundedness of $\left(v_{n}\left(y_{n}\right)\right)_{n=1}^{\infty}$, showing the uniform integrability of $\left(\frac{\mathrm{d} \mathbb{Q}_{n, y_{n}}^{r}}{\mathrm{~d} \mathbb{P}}\right)_{n=1}^{\infty}$.

To show that $\left(\frac{\mathrm{d} \mathbb{Q}_{n, y_{n}}^{r}}{\mathrm{dP}}\right)_{n=1}^{\infty}$ is Cauchy with respect to the topology of convergence in probability, suppose to the contrary that there is an $\alpha>0$ such that there are arbitrarily large $n$ and $m$ satisfying

$$
\mathbb{P}\left[\left|\frac{\mathrm{d} \mathbb{Q}_{n, y_{n}}^{r}}{\mathrm{~d} \mathbb{P}}-\frac{\mathrm{d} \mathbb{Q}_{m, y_{m}}^{r}}{\mathrm{~d} \mathbb{P}}\right|>\alpha\right]>\alpha .
$$

We now use the boundedness of $\left(v_{n}\left(y_{n}\right)\right)_{n=1}^{\infty}$ to find $N \in \mathbb{N}$ and a compact set $K$ contained in $\left\{y \geq 0: V_{N}(y)=V(y)<\infty\right\}$ such that, for $n \geq N$,

$$
\mathbb{P}\left[\frac{\mathrm{d} \mathbb{Q}_{n, y_{n}}}{\mathrm{~d} \mathbb{P}} \notin K\right]<\alpha / 3 \text {. }
$$

For suppose not, and suppose that $V(0)=\infty$. Choose $N \in \mathbb{N}$ such that

$$
\min \left\{V\left(U^{\prime}(N)\right), V\left(U^{\prime}(-N)\right)\right\}>\frac{3}{\alpha}\left(\sup _{n \in \mathbb{N}} v_{n}\left(y_{n}\right)+\sup _{n \in \mathbb{N}} y_{n}\left\|B^{+}\right\|_{\infty}\right) .
$$

Let $K_{N}$ denote the compact interval $\left[U^{\prime}(N), U^{\prime}(-N)\right]$. Then there exists $n \geq N$ such that

$$
\mathbb{P}\left[y_{n} \frac{\mathrm{d} \mathbb{Q}_{n, y_{n}}^{r}}{\mathrm{~d} \mathbb{P}} \notin K_{N}\right] \geq \frac{\alpha}{3}
$$

Now

$$
\begin{aligned}
v_{n}\left(y_{n}\right) & \geq \mathbb{E}\left[V_{n}\left(y_{n} \frac{\mathrm{d} \mathbb{Q}_{n, y_{n}}^{r}}{\mathrm{dP}}\right)\right]-y_{n}\left\|B^{+}\right\|_{\infty} \\
& \geq \mathbb{E}\left[V_{n}\left(y_{n} \frac{\mathrm{d} \mathbb{Q}_{n, y_{n}}^{r}}{\mathrm{dP}}\right) \chi_{\left\{y_{n} \frac{\left.\mathrm{d}_{n, y_{n}}^{r} \notin K_{N}\right\}}{\mathrm{d} \mathbb{P}} \notin K_{N}\right]-y_{n}\left\|B^{+}\right\|_{\infty}}\right. \\
& \geq \frac{\alpha}{3} \min \left\{V\left(U^{\prime}(N)\right), V\left(U^{\prime}(-N)\right)\right\}-y_{n}\left\|B^{+}\right\|_{\infty} \\
& >\sup _{n \in \mathbb{N}} v_{n}\left(y_{n}\right),
\end{aligned}
$$


which is a contradiction. In the case where $V(0)<\infty$, one can similarly find a compact set $K_{N}=\left[0, U^{\prime}(-N)\right]$ for which (24) holds.

Note that equations (23) and (24) imply that there are arbitrarily large $n$ and $m$ satisfying

$$
\mathbb{P}\left[\left|\frac{\mathrm{d} \mathbb{Q}_{n, y_{n}}^{r}}{\mathrm{~d} \mathbb{P}}-\frac{\mathrm{d} \mathbb{Q}_{m, y_{m}}^{r}}{\mathrm{~d} \mathbb{P}}\right|>\alpha \text { and } \frac{\mathrm{d} \mathbb{Q}_{n, y_{n}}}{\mathrm{~d} \mathbb{P}}, \frac{\mathrm{d} \mathbb{Q}_{m, y_{m}}}{\mathrm{~d} \mathbb{P}} \in K\right]>\alpha / 3 .
$$

By the strict convexity of $V$ and the compactness of $K$ we may find an $\eta>0$ such that, for $y_{1}, y_{2} \in K$ with $\left|y_{1}-y_{2}\right|>\alpha$, we have

$$
V\left(\frac{y_{1}+y_{2}}{2}\right) \leq \frac{V\left(y_{1}\right)+V\left(y_{2}\right)}{2}-\eta .
$$

Note that since $V$ is convex, this above inequality with $\eta=0$ is true for all other $y_{1}, y_{2}$.

Choose $\varepsilon>0$ so that

$$
\varepsilon \sup _{k} v_{k}\left(y_{k}\right)<\frac{\alpha \eta}{12} \text { and } \varepsilon\left\|B^{+}\right\|_{\infty} \sup _{k} y_{k}<\frac{\alpha \eta}{12} .
$$

Using [14], Corollary 4.2, there exists a constant $\delta$ such that for all $\lambda$ such that $(1-\delta)<\lambda<(1+\delta)$ and for all $n \in \mathbb{N}$ we have

$$
(1-\varepsilon) V_{n}(y)<V_{n}(\lambda y)<(1+\varepsilon) V_{n}(y) .
$$

Now choose $N$ so that for all $n \geq m \geq N$,

$$
\begin{aligned}
& 1-\delta \leq \frac{y_{m}}{y_{n}} \leq \min \{1+\delta, 1+\varepsilon\}, \\
& \left(y_{n}-y_{m}\right)\left\|B^{+}\right\|_{\infty}<\frac{\alpha \eta}{12}, \\
& v_{n}\left(y_{n}\right)-v_{m}\left(y_{m}\right)<\frac{\alpha \eta}{12} .
\end{aligned}
$$

Finally, choose $n \geq m \geq N$ so that (25) holds. It now follows that

$$
\begin{aligned}
v_{m}\left(y_{m}\right) \leq & \mathbb{E}\left[V_{m}\left(\frac{y_{m}}{2}\left(\frac{\mathrm{d} \mathbb{Q}_{n, y_{n}}^{r}}{\mathrm{~d} \mathbb{P}}+\frac{\mathrm{d} \mathbb{Q}_{m, y_{m}}^{r}}{\mathrm{~d} \mathbb{P}}\right)\right)\right] \\
& +y_{m}(m+1)\left(1-\frac{1}{2} \mathbb{E}\left[\frac{\mathrm{d} \mathbb{Q}_{n, y_{n}}^{r}}{\mathrm{~d} \mathbb{P}}+\frac{\mathrm{d} \mathbb{Q}_{m, y_{m}}^{r}}{\mathrm{~d} \mathbb{P}}\right]\right) \\
& -\frac{y_{m}}{2}\left\langle\mathbb{Q}_{n, y_{n}}+\mathbb{Q}_{m, y_{m}}, B\right\rangle \\
\leq & \frac{1}{2}\left[V_{m}\left(y_{m} \frac{\mathrm{d} \mathbb{Q}_{n, y_{n}}^{r}}{\mathrm{~d} \mathbb{P}}\right)\right]+\frac{1}{2} \mathbb{E}\left[V_{m}\left(y_{m} \frac{\mathrm{d} \mathbb{Q}_{m, y_{m}}^{r}}{\mathrm{~d} \mathbb{P}}\right)\right] \\
& -\eta \mathbb{P}\left[\left|\frac{\mathrm{d} \mathbb{Q}_{n, y_{n}}^{r}}{\mathrm{~d} \mathbb{P}}-\frac{\mathrm{d} \mathbb{Q}_{m, y_{m}}^{r}}{\mathrm{~d} \mathbb{P}}\right|>\alpha \text { and } \frac{\mathrm{d} \mathbb{Q}_{n, y_{n}}^{r}}{\mathrm{~d} \mathbb{P}}, \frac{\left.\mathrm{d} \mathbb{Q}_{m, y_{m}}^{r} \in K\right]}{\mathrm{d} \mathbb{P}} \in K\right.
\end{aligned}
$$




$$
\begin{aligned}
& +\frac{1}{2} y_{m}(m+1)\left(1-\mathbb{E}\left[\frac{\mathrm{d} \mathbb{Q}_{n, y_{n}}^{r}}{\mathrm{~d} \mathbb{P}}\right]\right)-\frac{1}{2} y_{m}\left\langle\mathbb{Q}_{n, y_{n}}, B\right\rangle \\
& +\frac{1}{2} y_{m}(m+1)\left(1-\mathbb{E}\left[\frac{\mathrm{d} \mathbb{Q}_{m, y_{m}}^{r}}{\mathrm{~d} \mathbb{P}}\right]\right)-\frac{1}{2} y_{m}\left\langle\mathbb{Q}_{m, y_{m}}, B\right\rangle \\
& \leq \frac{1}{2} \mathbb{E}\left[V_{n}\left(y_{m} \frac{\mathrm{d} \mathbb{Q}_{n, y_{n}}^{r}}{\mathrm{~d} \mathbb{P}}\right)\right]+\frac{1}{2} y_{m}(n+1)\left(1-\mathbb{E}\left[\frac{\mathrm{d} \mathbb{Q}_{n, y_{n}}^{r}}{\mathrm{~d} \mathbb{P}}\right]\right) \\
& -\frac{1}{2} y_{m}\left\langle\mathbb{Q}_{n, y_{n}}, B\right\rangle+\frac{1}{2} \mathbb{E}\left[V_{m}\left(y_{m} \frac{\mathrm{d} \mathbb{Q}_{m, y_{m}}^{r}}{\mathrm{~d} \mathbb{P}}\right)\right] \\
& +\frac{1}{2} y_{m}(m+1)\left(1-\mathbb{E}\left[\frac{\mathrm{d} \mathbb{Q}_{m, y_{m}}^{r}}{\mathrm{~d} \mathbb{P}}\right]\right)-\frac{1}{2} y_{m}\left\langle\mathbb{Q}_{m, y_{m}}, B\right\rangle-\frac{\alpha \eta}{3} \\
& \leq \frac{1}{2}(1+\varepsilon)\left\{\mathbb{E}\left[V_{n}\left(y_{n} \frac{\mathrm{d} \mathbb{Q}_{n, y_{n}}^{r}}{\mathrm{~d} \mathbb{P}}\right)\right]+y_{n}(n+1)\left(1-\mathbb{E}\left[\frac{\mathrm{d} \mathbb{Q}_{n, y_{n}}^{r}}{\mathrm{~d} \mathbb{P}}\right]\right)\right. \\
& \left.-y_{n}\left\langle\mathbb{Q}_{n, y_{n}}, B\right\rangle\right\}+\frac{1}{2}\left((1+\varepsilon) y_{n}-y_{m}\right)\left\|B^{+}\right\|_{\infty} \\
& +\frac{1}{2} \mathbb{E}\left[V_{m}\left(y_{m} \frac{\mathrm{d} \mathbb{Q}_{m, y_{m}}^{r}}{\mathrm{~d} \mathbb{P}}\right)\right]+\frac{1}{2} y_{m}(m+1)\left(1-\mathbb{E}\left[\frac{\mathrm{d} \mathbb{Q}_{m, y_{m}}^{r}}{\mathrm{~d} \mathbb{P}}\right]\right) \\
& -\frac{1}{2} y_{m}\left\langle\mathbb{Q}_{m, y_{m}}, B\right\rangle_{\infty}-\frac{\alpha \eta}{3} \\
& \leq \frac{1}{2}(1+\varepsilon) v_{n}\left(y_{n}\right)+\frac{1}{2} v_{m}\left(y_{m}\right)+\frac{1}{2}\left(y_{n}-y_{m}\right)\left\|B^{+}\right\|_{\infty} \\
& +\frac{1}{2} \varepsilon \sup _{k} y_{k}\left\|B^{+}\right\|_{\infty}-\frac{\alpha \eta}{3} \\
& \leq v_{m}\left(y_{m}\right)+\frac{1}{24} \alpha \eta+\frac{1}{24} \alpha \eta+\frac{1}{24} \alpha \eta+\frac{1}{24} \alpha \eta-\frac{\alpha \eta}{3} \text {. }
\end{aligned}
$$

This contradiction shows that $\left(\frac{\mathrm{d} \mathbb{Q}_{n, y_{n}}^{r}}{\mathrm{~d} \mathbb{P}}\right)_{n=1}^{\infty}$ is Cauchy in the topology of convergence in probability and therefore converges in the norm of $L^{1}(\mathbb{P})$ to a random variable which we denote by $\frac{d \mathbb{Q}_{y}}{d \mathbb{P}}$. The measure $\mathbb{Q}_{y}$ is by definition absolutely continuous with respect to $\mathbb{P}$. The fact that $\mathbb{Q}_{y}$ has mass 1 now follows since

$$
\mathbb{E}\left[\frac{\mathrm{d} \mathbb{Q}_{y}}{\mathrm{~d} \mathbb{P}}\right]=\lim _{n \rightarrow \infty} \mathbb{E}\left[\frac{\mathrm{d} \mathbb{Q}_{n, y_{n}}^{r}}{\mathrm{~d} \mathbb{P}}\right]=1
$$

For suppose that the second equality does not hold, that is,

$$
\liminf _{n \rightarrow \infty}\left[\frac{\mathrm{d} \mathbb{Q}_{n, y_{n}}^{r}}{\mathrm{~d} \mathbb{P}}\right]<1
$$


Then using Lemma 2.3 and equation (12),

$$
\begin{aligned}
v_{\infty}(y)= & \limsup _{n \rightarrow \infty} v_{n}\left(y_{n}\right) \\
= & \limsup _{n \rightarrow \infty}\left\{\mathbb{E}\left[V_{n}\left(y_{n} \frac{\mathrm{d} \mathbb{Q}_{n, y_{n}}^{r}}{\mathrm{~d} \mathbb{P}}\right)\right]+y_{n}(n+1)\left(1-\mathbb{E}\left[\frac{\mathrm{d} \mathbb{Q}_{n, y_{n}}^{r}}{\mathrm{~d} \mathbb{P}}\right]\right)\right. \\
& \left.-y_{n}\left\langle\mathbb{Q}_{n, y_{n}}, B\right\rangle\right\} \\
\geq & \limsup _{n \rightarrow \infty}\left\{0+y_{n}(n+1)\left(1-\mathbb{E}\left[\frac{\mathrm{d} \mathbb{Q}_{n, y_{n}}^{r}}{\mathrm{~d} \mathbb{P}}\right]\right)-y_{n}\left\|B^{+}\right\|_{\infty}\right\} \\
= &
\end{aligned}
$$

which is a contradiction. Note that an equivalent statement to (27) is that the singular parts of $\mathbb{Q}_{n, y_{n}}$ tend to 0 in the norm of $\left(L^{\infty}\right)^{*}$ as $n \rightarrow \infty$.

We finally prove that $\mathbb{Q}_{y} \in \mathcal{M}^{a}(S)$, using Remark 2.1(iii), that $D \cap L^{1}=\mathcal{M}^{a}(S)$ and $\mathscr{D}$ is weak ${ }^{*}$ compact. Since $\mathbb{Q}_{n, y_{n}} \stackrel{\left(L^{\infty}\right)^{*}}{\longrightarrow} \mathbb{Q}_{y}$, we have $\mathbb{Q}_{n, y_{n}} \stackrel{\text { weak }^{*}}{\longrightarrow} \mathbb{Q}_{y}$. Using the weak* closedness of $\mathscr{D}$, we see that $\mathbb{Q}_{y} \in \mathscr{D}$, and therefore $\mathbb{Q}_{y} \in \mathcal{M}^{a}(S)$.

ProOF OF COROLlary 3.2(i). Take a sequence $y_{k}$ tending to $y>0$. Using Lemma 3.1(i), we can find an increasing sequence $\left(n_{k}\right)_{k=1}^{\infty}$ such that

$$
\lim _{k \rightarrow \infty}\left\|\frac{\mathrm{d} \mathbb{Q}_{y_{k}}}{\mathrm{~d} \mathbb{P}}-\frac{\mathrm{d} \mathbb{Q}_{n_{k}, y_{k}}^{r}}{\mathrm{~d} \mathbb{P}}\right\|_{L^{1}(\mathbb{P})}=0
$$

Using Lemma 3.1(i) a second time, we see that

$$
\lim _{k \rightarrow \infty}\left\|\frac{\mathrm{d} \mathbb{Q}_{n_{k}, y_{k}}^{r}}{\mathrm{~d} \mathbb{P}}-\frac{\mathrm{d} \mathbb{Q}_{y}}{\mathrm{~d} \mathbb{P}}\right\|_{L^{1}(\mathbb{P})}=0 .
$$

Continuity of the map $y \mapsto \mathbb{Q}_{y}$ in the $L^{1}(\mathbb{P})$ norm follows from an application of the triangle inequality.

Using the fact that $\frac{d \mathbb{Q}_{n, y_{n}}^{r}}{\mathrm{~d} \mathbb{P}}$ converges in probability to $\frac{\mathrm{d} \mathbb{Q}_{y}}{\mathrm{dP}}$, we see that the random variables $V_{n}\left(y_{n} \frac{\mathrm{d} \mathbb{Q}_{n, y_{n}}^{r}}{\mathrm{~d} \mathbb{P}}\right)$ converge in probability to $V\left(y \frac{\mathrm{d} \mathbb{Q}_{y}}{\mathrm{dP}}\right)$. Using a version of Fatou's Lemma for limits in probability, followed by an application of equation (12), Lemma 2.3 and Lemma 2.2, we get

$$
\begin{aligned}
v(y) & \leq \mathbb{E}\left[V\left(y \frac{\mathrm{d} \mathbb{Q}_{y}}{\mathrm{~d} \mathbb{P}}\right)-y \frac{\mathrm{d} \mathbb{Q}_{y}}{\mathrm{~d} \mathbb{P}} B\right] \\
& \leq \liminf _{n \rightarrow \infty}\left\{\mathbb{E}\left[V_{n}\left(y_{n} \frac{\mathrm{d} \mathbb{Q}_{n, y_{n}}^{r}}{\mathrm{~d} \mathbb{P}}\right)\right]-y_{n}\left\langle\mathbb{Q}_{n, y_{n}}, B\right\rangle\right\} \\
& \leq \liminf _{n \rightarrow \infty} v_{n}\left(y_{n}\right)=v_{\infty}(y) \leq v(y) .
\end{aligned}
$$


We have in fact equality above, and thus $\mathbb{Q}_{y}$ is the minimizer of (6). The strict convexity of $v$ and uniqueness of $\mathbb{Q}_{y}$ follow from convexity of $\mathcal{M}^{a}(S)$, strict convexity of $V$ and formula (19).

PROOF OF LEMMA 3.1(ii). It follows easily from equation (28) that $V\left(\frac{\mathrm{d} \mathbb{Q}_{y}}{\mathrm{~d} \mathbb{P}}\right) \in L^{1}(\mathbb{P})$. Also, since $\left(V_{n}\left(y_{n} \frac{\mathrm{d} \mathbb{Q}_{n, y_{n}}^{r}}{\mathrm{~d} \mathbb{P}}\right)\right)_{n=1}^{\infty}$ converges in probability to $V\left(y \frac{\mathrm{d} \mathbb{Q}_{y}}{\mathrm{~d} \mathbb{P}}\right)$ and

$$
\liminf _{n \rightarrow \infty}\left[V_{n}\left(y_{n} \frac{\mathrm{d} \mathbb{Q}_{n, y_{n}}^{r}}{\mathrm{~d} \mathbb{P}}\right)\right]=\mathbb{E}\left[V\left(y \frac{\mathrm{d} \mathbb{Q}_{y}}{\mathrm{~d} \mathbb{P}}\right)\right]
$$

we must have convergence in the norm of $L^{1}(\mathbb{P})$.

Note for future use that we have simultaneously proved that

$$
\lim _{n \rightarrow \infty}(n+1)\left(1-\mathbb{E}\left[\frac{\mathrm{d} \mathbb{Q}_{n, y_{n}}^{r}}{\mathrm{~d} \mathbb{P}}\right]\right)=0 .
$$

Proof OF COROLlary 3.2(ii). Similar to the proof of Corollary 3.2(i). Follows from the convergence of $\left(V_{n}\left(y_{n} \frac{\mathrm{d} \mathbb{Q}_{n, y_{n}}^{r}}{\mathrm{~d} \mathbb{P}}\right)\right)_{n=1}^{\infty}$ in the $L^{1}(\mathbb{P})$ norm.

Proof of Lemma 3.1(iii). By [14], Corollary 4.2(ii) there is a constant $C$ such that

$$
y\left|V_{n}^{\prime}(y)\right| \leq C V_{n}(y) \quad \text { for } y \geq 0,
$$

uniformly in $n \in \mathbb{N}$, where, in the case $y=0$ we adopt the rule $0 \cdot \infty=0$. Hence the sequence of random variables $\left(y_{n} \frac{\mathrm{d} \mathbb{Q}_{n, y_{n}}^{r}}{\mathrm{~d} \mathbb{P}} V_{n}^{\prime}\left(y_{n} \frac{\mathrm{d} \mathbb{Q}_{n, y_{n}}^{r}}{\mathrm{~d} \mathbb{P}}\right)\right)_{n=1}^{\infty}$ is dominated in absolute value by the $L^{1}$-convergent sequence $\left(C V_{n}\left(y_{n} \frac{\mathrm{d} \mathbb{Q}_{n, y_{n}}^{r}}{\mathrm{~d} \mathbb{P}}\right)\right)_{n=1}^{\infty}$ and is therefore uniformly integrable. By the continuity of the map $y \mapsto y V^{\prime}(y)$, which holds true, for $y>0$, and, in the case $V(0)=U(\infty)<\infty$, for $y \geq 0$ too, we also have that $\left(y_{n} \frac{\mathrm{d} \mathbb{Q}_{n, y_{n}}^{r}}{\mathrm{~d} \mathbb{P}} V_{n}^{\prime}\left(y_{n} \frac{\mathrm{d} \mathbb{Q}_{n, y_{n}}^{r}}{\mathrm{~d} \mathbb{P}}\right)\right)_{n=1}^{\infty}$ converges in probability to $y \frac{\mathrm{d} \mathbb{Q}_{y}}{\mathrm{~d} \mathbb{P}} V^{\prime}\left(y \frac{\mathrm{d} \mathbb{Q}_{y}}{\mathrm{~d} \mathbb{P}}\right)$, and therefore converges in the norm of $L^{1}(\mathbb{P})$.

PROOF OF COROLlaRY 3.2(iii). The assertion of continuity with respect to the $L^{1}(\mathbb{P})$ norm now follows by the same easy argument as at the end of Corollary 3.2(i) from the convergence of $\left(y_{n} \frac{\mathrm{d}_{\mathbb{Q}_{n, y_{n}}}}{\mathrm{~d} \mathbb{P}} V_{n}^{\prime}\left(y_{n} \frac{\mathrm{d} \mathbb{Q}_{n, y_{n}}^{r}}{\mathrm{~d} \mathbb{P}}\right)\right)_{n=1}^{\infty}$ in the $L^{1}(\mathbb{P})$ norm. To prove formula (20), first observe that the term on the right hand side is a continuous function of $y>0$. Existence of the derivative $v^{\prime}(y)$ is guaranteed for all but countably many $y$ 's by the convexity of $v$. It will suffice, therefore, to show (20) wherever the derivative $v^{\prime}(y)$ exists. Let $\left(y_{n}\right)_{n=1}^{\infty}$ be a sequence tending 
to $y>0$ such that $v^{\prime}(y)$ exists. Using Lemma 2.3, equation (15), Lemma 3.1(iii), equation (29) and Lemma 3.1(i) we see that

$$
\begin{aligned}
& v^{\prime}(y)= \lim _{n \rightarrow \infty} v_{n}^{\prime}\left(y_{n}\right) \\
&= \lim _{n \rightarrow \infty}\left\{\mathbb{E}\left[\frac{\mathrm{d} \mathbb{Q}_{n, y_{n}}^{r}}{\mathrm{~d} \mathbb{P}} V_{n}^{\prime}\left(y_{n} \frac{\mathrm{d} \mathbb{Q}_{n, y_{n}}^{r}}{\mathrm{~d} \mathbb{P}}\right)\right]+(n+1)\left(1-\mathbb{E}\left[\frac{\mathrm{d} \mathbb{Q}_{n, y_{n}}^{r}}{\mathrm{~d} \mathbb{P}}\right]\right)\right. \\
&\left.-\left\langle\mathbb{Q}_{n, y_{n}}, B\right\rangle\right\} \\
&=\mathbb{E}\left[\frac{\mathrm{d} \mathbb{Q}_{y}}{\mathrm{~d} \mathbb{P}} V^{\prime}\left(y \frac{\mathrm{d} \mathbb{Q}_{y}}{\mathrm{~d} \mathbb{P}}\right)-\frac{\mathrm{d} \mathbb{Q}_{y}}{\mathrm{~d} \mathbb{P}} B\right] .
\end{aligned}
$$

PROOF OF LEMMA 3.1(iv). The convergence in probability of the sequence $\left(y \frac{\mathrm{d}_{y}}{\mathrm{~d} \mathbb{P}} V_{n}^{\prime}\left(y_{n} \frac{\mathrm{d} \mathbb{Q}_{n, y_{n}}^{r}}{\mathrm{~d} \mathbb{P}}\right)\right)_{n=1}^{\infty}$ towards $y \frac{\mathrm{d}_{y}}{\mathrm{dP}} V^{\prime}\left(y \frac{\mathrm{d} \mathbb{Q}_{y}}{\mathrm{dP}}\right)$ is obvious. We now prove uniform integrability of the positive parts:

$$
\begin{aligned}
& y \frac{\mathrm{d} \mathbb{Q}_{y}}{\mathrm{~d} \mathbb{P}} V_{n}^{\prime}\left(y_{n} \frac{\mathrm{d} \mathbb{Q}_{n, y_{n}}^{r}}{\mathrm{~d} \mathbb{P}}\right)^{+}
\end{aligned}
$$

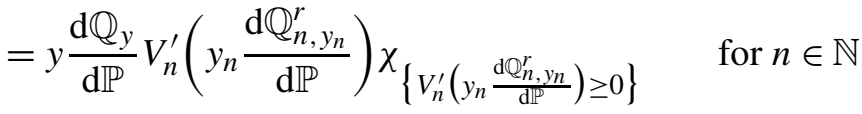

$$
\begin{aligned}
& \leq \max \left\{y \frac{\mathrm{d} \mathbb{Q}_{y}}{\mathrm{~d} \mathbb{P}} V^{\prime}\left(y \frac{\mathrm{d} \mathbb{Q}_{y}}{\mathrm{~d} \mathbb{P}}\right) \chi_{\left\{V_{n}^{\prime}\left(y_{n} \frac{\left.\left.\mathrm{d} \mathbb{Q}_{n, y_{n}}^{r}\right) \geq 0\right\}}{\mathrm{d} \mathbb{P}}\right)\right.},\right. \\
& \left.y_{n} \frac{\mathrm{d} \mathbb{Q}_{n, y_{n}}^{r}}{\mathrm{~d} \mathbb{P}} V_{n}^{\prime}\left(y_{n} \frac{\mathrm{d} \mathbb{Q}_{n, y_{n}}}{\mathrm{~d} \mathbb{P}}\right) \chi_{\left\{V_{n}^{\prime}\left(y_{n} \frac{\left.\left.\mathbb{d}_{n, y_{n}}^{r}\right) \geq 0\right\}}{\mathrm{d} \mathbb{P}}\right)\right.}\right\}
\end{aligned}
$$

which is easily verified by distinguishing pointwise the cases $y \frac{\mathrm{d} \mathbb{Q}_{y}}{\mathrm{~d} \mathbb{P}} \geq y_{n} \frac{\mathrm{d} \mathbb{Q}_{n, y_{n}}^{r}}{\mathrm{~d} \mathbb{P}}$ and $y \frac{\mathrm{d}_{y}}{\mathrm{dP}}<y_{n} \frac{\mathrm{d} \mathbb{Q}_{n, y_{n}}^{r}}{\mathrm{dP}}$. As the family of functions on the right hand side of (32) is uniformly integrable we have proved the uniform integrability of the positive parts of the sequence.

Let $x_{n}:=-v_{n}^{\prime}\left(y_{n}\right)$. Using Lemma 2.3 we have $\lim _{n \rightarrow \infty} x_{n}=-v^{\prime}(y)$. Observe from equations (16) and (14) that

$$
\mathbb{E}\left[x_{n}+\frac{\mathrm{d} \mathbb{Q}_{y}}{\mathrm{~d} \mathbb{P}} V_{n}^{\prime}\left(y_{n} \frac{\mathrm{d} \mathbb{Q}_{n, y_{n}}^{r}}{\mathrm{~d} \mathbb{P}}\right)-\frac{\mathrm{d} \mathbb{Q}_{y}}{\mathrm{~d} \mathbb{P}} B\right]=-\mathbb{E}\left[\frac{\mathrm{d} \mathbb{Q}_{y}}{\mathrm{~d} \mathbb{P}} \hat{X}^{n}\left(x_{n}\right)\right] \geq 0
$$

because $\mathbb{Q}_{y} \in \mathscr{D}$ and $\hat{X}^{n}\left(x_{n}\right) \in \mathcal{X}_{0}$. Hence using the formula for $v^{\prime}(y)$ from Corollary 3.2(iii),

$$
\begin{aligned}
\liminf _{n \rightarrow \infty} & \mathbb{E}\left[y \frac{\mathrm{d} \mathbb{Q}_{y}}{\mathrm{~d} \mathbb{P}} V_{n}^{\prime}\left(y_{n} \frac{\mathrm{d} \mathbb{Q}_{n, y_{n}}^{r}}{\mathrm{~d} \mathbb{P}}\right)\right] \\
\geq & -x y+\mathbb{E}\left[y \frac{\mathrm{d} \mathbb{Q}_{y}}{\mathrm{~d} \mathbb{P}} B\right] \geq v^{\prime}(y) y+\mathbb{E}\left[y \frac{\mathrm{d} \mathbb{Q}_{y}}{\mathrm{~d} \mathbb{P}} B\right]=\mathbb{E}\left[y \frac{\mathrm{d} \mathbb{Q}_{y}}{\mathrm{~d} \mathbb{P}} V^{\prime}\left(y \frac{\mathrm{d} \mathbb{Q}_{y}}{\mathrm{~d} \mathbb{P}}\right)\right] .
\end{aligned}
$$


The result now follows because we have shown convergence in probability, uniform integrability of the positive parts and the above inequality satisfied by the expectations.

Proof OF Corollary 3.2(iv). Fix $x \in \mathbb{R}$ and let $y_{n}=u_{n}^{\prime}(x)$ as in equation (14). Now $u_{n} \nearrow u_{\infty} \leq u$ where $u_{\infty}$ is conjugate to $v_{\infty}=v$. Since $v$ is strictly convex, $u_{\infty}$ is continuously differentiable, and using Lemma 2.3, we see that $y_{n}=u_{n}^{\prime}(x) \rightarrow u_{\infty}^{\prime}(x)=y$ as $n \rightarrow \infty$. Using equation (14),

$$
\left\|\hat{X}(x)-\hat{X}_{T}^{n}(x)\right\|_{L^{1}(\mathbb{Q})}=\left\|\frac{\mathrm{d} \mathbb{Q}_{y}}{\mathrm{~d} \mathbb{P}} V^{\prime}\left(y \frac{\mathrm{d} \mathbb{Q}_{y}}{\mathrm{~d} \mathbb{P}}\right)-\frac{\mathrm{d} \mathbb{Q}_{y}}{\mathrm{~d} \mathbb{P}} V_{n}^{\prime}\left(y_{n} \frac{\mathrm{d} \mathbb{Q}_{n, y_{n}}^{r}}{\mathrm{~d} \mathbb{P}}\right)\right\|_{L^{1}(\mathbb{P})} \rightarrow 0
$$

as $n \rightarrow \infty$.

Proof OF LEMMA 3.1(v). We first show that

$$
\lim _{n \rightarrow \infty}\left\|U\left(x+\hat{X}^{n}(x)-B\right)-U_{n}\left(x+\hat{X}^{n}(x)-B\right)\right\|_{L^{1}(\mathbb{P})}=0 .
$$

Indeed, since $U_{n+1}(x)$ coincides with $U(x)$ for $x \geq-(n+1)$ and $x+\hat{X}^{n}(x)-B \geq$ $-(n+1)$ we have

$$
\begin{aligned}
& \mathbb{E}\left[U\left(x+\hat{X}^{n}(x)-B\right)\right]-\mathbb{E}\left[U_{n}\left(x+\hat{X}^{n}(x)-B\right)\right] \\
& \quad=\mathbb{E}\left[U_{n+1}\left(x+\hat{X}^{n}(x)-B\right)\right]-\mathbb{E}\left[U_{n}\left(x+\hat{X}^{n}(x)-B\right)\right] \\
& \quad \leq u_{n+1}(x)-u_{n}(x) .
\end{aligned}
$$

The right hand side of this last inequality tends to zero because the increasing sequence $\left(u_{n}(x)\right)_{n=1}^{\infty}$ is convergent.

Let $y_{n}=u_{n}^{\prime}(x)$. From equation (14) and Lemma 3.1 (ii) and (iii) we see that,

$$
\begin{aligned}
&\left\|U_{n}\left(x+\hat{X}^{n}(x)-B\right)-U_{m}\left(x+\hat{X}^{m}(x)-B\right)\right\|_{L^{1}(\mathbb{P})} \\
&=\| U_{n}\left(-V_{n}^{\prime}\left(y_{n} \frac{\left.\left.\mathrm{d} \mathbb{Q}_{n, y_{n}}^{r}\right)\right)-U_{m}\left(-V_{m}^{\prime}\left(y_{m} \frac{\mathrm{d} \mathbb{Q}_{m, y_{m}}^{r}}{\mathrm{~d} \mathbb{P}}\right)\right) \|_{L^{1}(\mathbb{P})}}{\leq} \| U_{n}\left(-V_{n}^{\prime}\left(y_{n} \frac{\mathrm{d} \mathbb{Q}_{n, y_{n}}^{r}}{\mathrm{~d} \mathbb{P}}\right)\right)+y_{n} \frac{\mathrm{d} \mathbb{Q}_{n, y_{n}}^{r}}{\mathrm{~d} \mathbb{P}} V_{n}^{\prime}\left(y_{n} \frac{\mathrm{d} \mathbb{Q}_{n, y_{n}}^{r}}{\mathrm{~d} \mathbb{P}}\right)\right.\right. \\
&-\left(U_{m}\left(-V_{m}^{\prime}\left(y_{m} \frac{\mathrm{d} \mathbb{Q}_{m, y_{m}}^{r}}{\mathrm{~d} \mathbb{P}}\right)\right)+y_{m} \frac{\mathrm{d} \mathbb{Q}_{m, y_{m}}^{r}}{\mathrm{~d} \mathbb{P}} V_{m}^{\prime}\left(y_{m} \frac{\mathrm{d} \mathbb{Q}_{m, y_{m}}^{r}}{\mathrm{~d} \mathbb{P}}\right)\right) \|_{L^{1}(\mathbb{P})} \\
&+\left\|y_{n} \frac{\mathrm{d} \mathbb{Q}_{n, y_{n}}^{r}}{\mathrm{~d} \mathbb{P}} V_{n}^{\prime}\left(y_{n} \frac{\mathrm{d} \mathbb{Q}_{n, y_{n}}^{r}}{\mathrm{~d} \mathbb{P}}\right)-y_{m} \frac{\mathrm{d} \mathbb{Q}_{m, y_{m}}^{r}}{\mathrm{~d} \mathbb{P}} V_{m}^{\prime}\left(y_{m} \frac{\mathrm{d} \mathbb{Q}_{m, y_{m}}^{r}}{\mathrm{~d} \mathbb{P}}\right)\right\|_{L^{1}(\mathbb{P})} \\
&=\left\|V_{n}\left(y_{n} \frac{\mathrm{d} \mathbb{Q}_{n, y_{n}}^{r}}{\mathrm{~d} \mathbb{P}}\right)-V_{m}\left(y_{m} \frac{\mathrm{d} \mathbb{Q}_{m, y_{m}}^{r}}{\mathrm{~d} \mathbb{P}}\right)\right\|_{L^{1}(\mathbb{P})}
\end{aligned}
$$




$$
\begin{aligned}
& +\left\|y_{n} \frac{\mathrm{d} \mathbb{Q}_{n, y_{n}}^{r}}{\mathrm{~d} \mathbb{P}} V_{n}^{\prime}\left(y_{n} \frac{\mathrm{d} \mathbb{Q}_{n, y_{n}}^{r}}{\mathrm{~d} \mathbb{P}}\right)-y_{m} \frac{\mathrm{d} \mathbb{Q}_{m, y_{m}}^{r}}{\mathrm{~d} \mathbb{P}} V_{m}^{\prime}\left(y_{m} \frac{\mathrm{d} \mathbb{Q}_{m, y_{m}}^{r}}{\mathrm{~d} \mathbb{P}}\right)\right\|_{L^{1}(\mathbb{P})} \\
\rightarrow & 0
\end{aligned}
$$

as $m, n \rightarrow \infty$. Hence

$$
\begin{aligned}
\| U(x+ & \left.\hat{X}^{n}(x)-B\right)-U\left(x+\hat{X}^{m}(x)-B\right) \|_{L^{1}(\mathbb{P})} \\
\leq & \left\|U_{n}\left(x+\hat{X}^{n}(x)-B\right)-U_{m}\left(x+\hat{X}^{m}(x)-B\right)\right\|_{L^{1}(\mathbb{P})} \\
& +\left\|U\left(x+\hat{X}^{m}(x)-B\right)-U_{m}\left(x+\hat{X}^{m}(x)-B\right)\right\|_{L^{1}(\mathbb{P})} \\
& +\left\|U\left(x+\hat{X}^{n}(x)-B\right)-U_{n}\left(x+\hat{X}^{n}(x)-B\right)\right\|_{L^{1}(\mathbb{P})} \\
& \rightarrow 0
\end{aligned}
$$

as $m, n \rightarrow \infty$. This implies that $\left(U\left(x+\hat{X}^{n}(x)-B\right)\right)_{n=1}^{\infty}$ is $L^{1}(\mathbb{P})$ convergent, and since it converges in probability to $U(x+\hat{X}(x)-B)$, we have proved (18). The random variable $\hat{X}(x)$ is in $\mathcal{X}_{U}(x)$ by the very definition of this set.

Proof OF COROLlary 3.2(v). Using Lemma 3.1(v) we see that $u_{\infty}(x)=$ $\mathbb{E}[U(x+\hat{X}(x)-B)]$. A classical calculation shows that

$$
\mathbb{E}[U(x+X-B)] \leq \mathbb{E}\left[V\left(y \frac{\mathrm{d} \mathbb{Q}}{\mathrm{dP}}\right)-y \frac{\mathrm{d} \mathbb{Q}}{\mathrm{dP}} B\right]
$$

for all $X \in \mathcal{X}_{U}(x)$ and all $y \in \mathbb{R}$ and all $\mathbb{Q} \in \mathcal{M}^{a}(S)$. See, for example, [1], Section 4. Since we have equality above for $X=\hat{X}(x), y=u^{\prime}(x)$ and $\mathbb{Q}=\mathbb{Q}_{y}$, we have that $\mathbb{E}[U(x+\hat{X}(x)-B)]=u(x)$. Thus $u=u_{\infty}$ and $u$ is conjugate to $v$.

To see the identity (21), note that

$$
u^{\prime}(x)=\mathbb{E}\left[y \frac{\mathrm{d} \mathbb{Q}_{y}}{\mathrm{~d} \mathbb{P}}\right]=\mathbb{E}\left[U^{\prime}\left(-V^{\prime}\left(y \frac{\mathrm{d} \mathbb{Q}_{y}}{\mathrm{~d} \mathbb{P}}\right)\right)\right]=\mathbb{E}\left[U^{\prime}(x+\hat{X}(x)-B)\right]
$$

The identity (22) is a reformulation of equation (20).

Using [14], Corollary 4.2, we have that, for each $\lambda>0$, there is a constant $C>0$ such that

$$
v(\lambda y)=\mathbb{E}\left[V\left(\lambda y \frac{\mathrm{d} \mathbb{Q}_{\lambda y}}{\mathrm{~d} \mathbb{P}}\right)\right] \leq \mathbb{E}\left[V\left(\lambda y \frac{\mathrm{d} \mathbb{Q}_{y}}{\mathrm{dP}}\right)\right] \leq C \mathbb{E}\left[V\left(y \frac{\mathrm{d} \mathbb{Q}_{y}}{\mathrm{dP}}\right)\right]=C v(y) .
$$

If follows from [14], Proposition 4.1 and [10], Corollary 6.1, that $u$ has reasonable asymptotic elasticity.

Suppose for a contradiction that $u^{\prime}(-\infty)=\alpha<\infty$. Then for all $\varepsilon>0$ there exists an $x_{0}$ such that for all $x \leq x_{0}$ we have $\alpha-\varepsilon \leq u^{\prime}(x) \leq \alpha$. It follows that for $x \leq x_{0}$,

$$
\operatorname{AE}_{-\infty}(u) \leq \liminf _{x \rightarrow-\infty} \frac{x u^{\prime}(x)}{u(x)} \leq \liminf _{x \rightarrow-\infty} \frac{\alpha x}{u\left(x_{0}\right)+(\alpha-\varepsilon)\left(x-x_{0}\right)}=\frac{\alpha}{\alpha-\varepsilon} .
$$


Since this is true for all $\varepsilon>0$ we have $\operatorname{AE}_{-\infty}(u)=1$, which is the desired contradiction.

Suppose for a contradiction that $u^{\prime}(\infty)=\alpha>0$. Then for all $\varepsilon>0$ there exists an $x_{0}$ such that for all $x \geq x_{0}$ we have $\alpha \leq u^{\prime}(x) \leq \alpha+\varepsilon$. It follows that for $x \geq x_{0}$

$$
\operatorname{AE}_{+\infty}(u) \geq \limsup _{x \rightarrow \infty} \frac{x u^{\prime}(x)}{u(x)} \geq \limsup _{x \rightarrow \infty} \frac{\alpha x}{u\left(x_{0}\right)+(\alpha+\varepsilon)\left(x-x_{0}\right)}=\frac{\alpha}{(\alpha+\varepsilon)} .
$$

Since this is true for all $\varepsilon>0$ we have $\operatorname{AE}_{\infty}(u)=1$, which is the desired contradiction.

Lemma 3.3. Assume that $\mathbb{Q}_{y} \sim \mathbb{P}$ and let $y=u^{\prime}(x)$. There exists a $\mathbb{Q}_{y}$-martingale

$$
\hat{X}_{t}(x)=(\hat{H} \cdot S)_{t}=\int_{0}^{t} \hat{H}_{u} \mathrm{~d} S_{u}, \quad 0 \leq t \leq T,
$$

where $\hat{H}_{t}=\hat{H}_{t}(x)$ is a predictable, S-integrable process and such that $\hat{X}_{T}(x)=$ $\hat{X}(x)$.

ProOF. From Lemma 3.1(iii) and the fact that $v$ is finitely valued we see that,

$$
\frac{\mathrm{d} \mathbb{Q}_{y}}{\mathrm{~d} \mathbb{P}} V^{\prime}\left(y \frac{\mathrm{d} \mathbb{Q}_{y}}{\mathrm{~d} \mathbb{P}}\right)-\frac{\mathrm{d} \mathbb{Q}_{y}}{\mathrm{~d} \mathbb{P}} B \in L^{1}(\mathbb{P}),
$$

and therefore the process

$$
\hat{X}_{t}(x):=-\mathbb{E}_{\mathbb{Q}_{y}}\left[x+V^{\prime}\left(y \frac{\mathrm{d} \mathbb{Q}_{y}}{\mathrm{~d} \mathbb{P}}\right)-B \mid \widetilde{F}_{t}\right]
$$

is a well-defined $\mathbb{Q}_{y}$-martingale. Note also from equation (20) that $\hat{X}_{0}(x)=0$.

From [1], Theorem 3.1, we see that each $\hat{X}^{n}(x)$ is a stochastic integral on $S$ starting at $\hat{X}_{0}^{n}(x)=0$ for some integrand $\hat{H}^{n}(x)$. In other words,

$$
\hat{X}_{t}^{n}=\left(\hat{H}^{n}(x) \cdot S\right)_{t}
$$

for $0 \leq t \leq T$. Note also, by Corollary 3.2(iv), that $\hat{X}_{T}^{n}(x) \rightarrow \hat{X}_{T}(x)$ in the norm of $L^{1}\left(\mathbb{Q}_{y}\right)$.

The rest of this proof is more or less identical to the proof of Step 10 of [14]. Following the proof therein, one can show that the limit of (34) as $n \rightarrow \infty$ is $\hat{X}_{t}=(\hat{H}(x) \cdot S)_{t}$, where $\hat{H}_{t}(x)$ is a predictable, $S$-integrable process.

Acknowledgments. I wish to thank Josef Teichmann, Elisa Nicolato, Friedrich Hubalek and Walter Schachermayer for many very helpful discussions which resulted in improvements and alterations to the article. 


\section{REFERENCES}

[1] Cvitanić, J., Schachermayer, W. and Wang, H. (2001). Utility maximisation in incomplete markets with random endowment. Finance and Stochastics 5 259-272.

[2] Davis, M. H. A. (1997). Option pricing in incomplete markets. In Mathematics of Derivative Securities (M. A. H. Dempster and S. R. Pliska, eds.) 216-226. Cambridge Univ. Press.

[3] Delbaen, F., Grandits, P., Rheinländer, T., Samperi, D., Schweizer, M. and STRICKER, C. (2001). Exponential hedging and entropic penalties. Math. Finance. To appear.

[4] Delbaen, F. and Schachermayer, W. (1994). A general version of the fundamental theorem of asset pricing. Math. Ann. 300 463-520.

[5] Frittelli, M. (2000). Optimal solutions to utility maximization and to the dual problem. Preprint.

[6] FREY, R. and Sin, C. A. (1999). Bounds on European option prices under stochastic volatility. Math. Finance 9 97-116.

[7] El Karoui, N. and QueneZ, M.-C. (1995). Dynamic programming and pricing of contingent claims in an incomplete market. SIAM J. Control Optim. 33 (1995) 29-66.

[8] El Karoui, N. and Rouge, R. (2000). Pricing via utility maximization and entropy. Math. Finance 10 259-276.

[9] KRAMKOV, D. O. (1996). Optional decomposition of supermartingales and hedging contingent claims in incomplete security markets. Probab. Theory Related Fields 105 459-479.

[10] Kramkov, D. and Schachermayer, W. (1999). The asymptotic elasticity of utility functions and optimal investment in incomplete markets. Ann. Appl. Probab. 9 904-950.

[11] LAng, S. (1993). Real and Functional Analysis, 3rd ed. Springer, New York.

[12] Rockafellar, R. T. (1970). Convex Analysis. Princeton Univ. Press.

[13] Schachermayer, W. (2000). Optimal investment in incomplete financial markets. In Proceedings of the First World Congress of the Bachelier Society (Paris). To appear.

[14] SchachermaYer, W. (2001). Optimal investment in incomplete markets when wealth may become negative. Ann. Appl. Probab. 11 694-734.

DEPARTMENT OF FINANCIAL AND

ACtUARIal Mathematics (E1075)

VIENNA UNIVERSITY OF TECHNOLOGY

WIEDNER HAUPTSTRASSE 8-10

1040 VIENNA

AUSTRIA

E-MAIL: mowen@fam.tuwien.ac.at 